\title{
Improvement of Leach Protocol with K Angle Optimization using an Optimized Algorithm in Wireless Sensor Networks
}

\author{
Ravneet kaur \\ Department of electronics and communication \\ Lovely professional university \\ Phagwara, India
}

\author{
Deepika Sharma \\ Assistant Professor in Department of Electronics and \\ communication \\ Lovely Professional University \\ Phagwara, India
}

\begin{abstract}
In this paper we are implementing an advanced optimizes clustering algorithm to enhance network lifetime when LEACH protocol is used. Main concept behind the wireless sensors network is to save energy more and more so that it works last long enough. This is due to fact that the size of a sensor node is expected to be small and this leads to constraints on size of its components i.e. battery size, processors, data storing memory, all are needed to be small. So any optimization in these networks should focus on optimizing energy consumption to enhance WSN life time. For this various protocols and algorithms by various approaches are there to enhance WSN lifetime. One of the approaches is to optimize angle by which we virtually split sensors with grid. In various leach protocols we use circular grid by taking sink at the center of the network. But we cannot optimize angle by which we make grid, here in this paper we are going to implement a new approach to optimize angle with using an optimized algorithm to get better cluster formation and enhances our results. Algorithm used in the proposed scheme is GENETIC ALGORITHM (GA).
\end{abstract}

\section{Keywords:}

Wireless Sensor Network, LEACH Protocol, GA.

\section{INTRODUCTION}

Wireless Sensor Networks (WSNs) consist of the number of sensor devices. The collection of the hundreds or thousands of sensor nodes deployed over a large geographical area the parameter of which has to be measured. These sensors nodes sense the data by using their sensors from remote locations by interacting with physical environment and relaying the information by coordinating among the sensors. The sensor node consists of an embedded microprocessor, radio transceiver for communication, memory storage that is limited and on-board sensors for sensing the parameters such as temperature, humidity, pressure etc. The sensor nodes collect the data from the sensing field and send the data to the base station (BS). The ability of WSN to communicate the environmental data to base station directly or through neighboring node makes the sensor network suitable for remote control monitoring and surveillance [1].
The nodes having with typically low mobility and they are limited in capabilities like energy supply and bandwidth. The lifetime of sensor networks should be as long as possible. On the other hand the battery recharging in many cases may be inconvenient or impossible [2]. Even though the nodes in sensor network may have same initial energy, but the energy of each node will not be same after transmission of data among the nodes. This is all due to the radio communication characteristics of wireless sensor networks (WSN). A smart way to prolong the lifetime of sensor networks and sensor nodes is to make efficient use of energy. Many energy efficient algorithms and energy efficient protocols have been proposed for WSN [3].

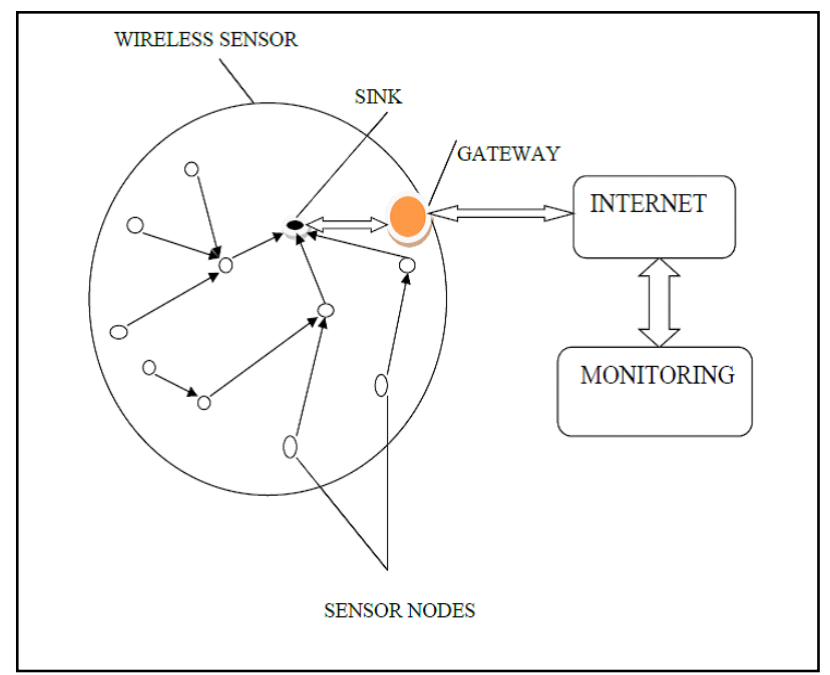

Fig.1. Wireless sensor network

The most commonly used protocol which is proved energy efficient is the LEACH protocol. In this protocol, to guarantee low energy consumption and for uniform load distribution on the sensor nodes over the network and the sensor nodes are organized into clusters [1]. 


\section{LEACH PROTOCOL}

Leach is "Energy Efficient Adaptive Protocol for Cluster Hierarchy in Wireless Sensor Networks". This protocol works on the principal that the node with more residual energy having with more chances to be elected as a cluster head in a particular cluster. The energy load should be evenly distributed among all the cluster members of the particular cluster in order improve the life time of a wireless sensor network. By doing so the energy at a single node or a small set of nodes will not drained out soon. LEACH is a first energy efficient routing protocol for hierarchical clustering. By implementing LEACH protocol on a sensor network we can make the network more energy efficient. LEACH protocol divides the nodes in a sensor network in the different clusters. Then one node from each cluster is elected as a cluster head $(\mathrm{CH})$ and the other nodes in the clusters are known as cluster members (CM). Cluster member nodes sense the sensing field and get the data. Then the sensed data is transmitted to the cluster heads by the cluster members. The cluster head receives the data and aggregate the data. Now this aggregated data is transmitted to the sink node by the cluster heads.

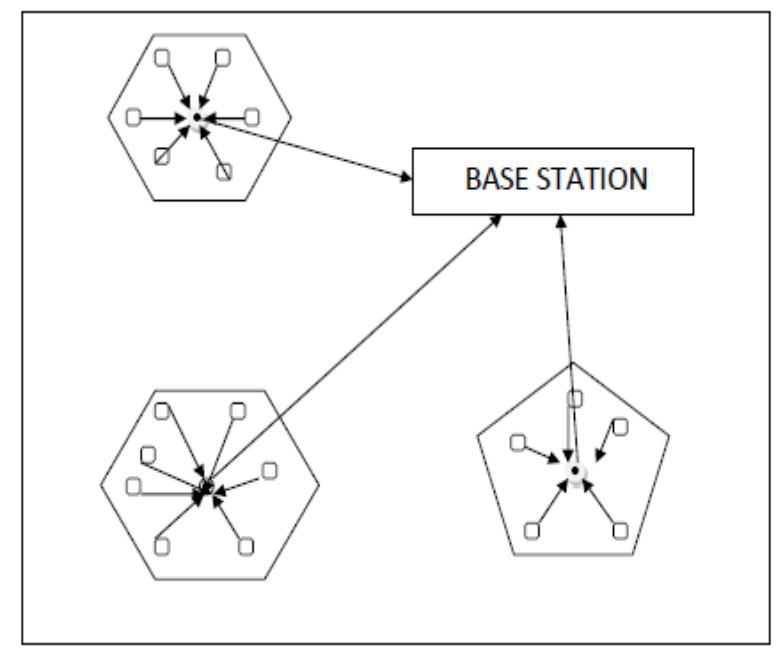

Fig.2. LEACH Protocol Architecture

There are mainly two phases in LEACH protocol one is Setup phase and other is Steady-state phase. In the first phase that is setup phase nodes in a sensor field are divided into clusters and then the cluster head is elected from these nodes. In steady-state phase, the data is transmitted from cluster member nodes to the cluster head and then again data is transmitted from cluster head to sink node or base station. TDMA scheme that is time division multiple access is used for the efficient communication between the sensor nodes. A particular time slot is provided to the cluster members for transmission of data, in this time slot cluster members can transmit data to the cluster head. This scheme is used to avoid collision that occurs during data transmission. The selection of cluster head is takes place randomly after every round [4] [5] [6].

\section{GENETIC ALGORITHM}

Genetic algorithm is a method for the optimization and global research. A Genetic Algorithm performs fitness tests to select the best population from the new structure. The quality of the individual is determined by fitness on the basis of the criteria that is defined. The ability of an individual to pass on its genetic material is called as fitness of that individual. Anything that contributes to this ability will also contribute to the organism of overall fitness. This ability of an individual includes the facts that enable it to survive and also further reproduction should be possible. The function that defines the problem evaluates the fitness value in GA. Fate of an individual also depends on fitness value. More the fitness value will lead to the more chances of that individual to survive. GA is mainly used to solve those problems whose mathematical solutions are not possible. For such problems GA provides an optimum solution. Basically process of GA includes five steps. These steps are population, fitness value, selection, crossover and mutation. These steps are described below: 1) Population: A population mainly consists of a group of individuals. These individuals are called as chromosomes and represent the complete solution of a problem which is defined. Each chromosome is represented with a sequence of 0 s or $1 \mathrm{~s}$. By randomly generating the set of individuals, a set of population can be created. 2) Fitness: The ability of an individual to pass on its genetic material is defined as the fitness value of that individual. Fate of an individual also depends on fitness value. More the fitness value will lead to the more chances of that individual to survive. In GA the function that defines the problem is used to evaluate the fitness value.3) Selection: The selection process used to determine or used to select the chromosomes from the current population that will mate (crossover) to create new population with new chromosomes. The individuals with the better fitness values will have better chances of selection. 4) Crossover: Recombination of component materials due to mating is known as cross over. It is responsible for the transfer of genetic inheritance. The outcome of crossover depends on the selection of chromosomes from population as performed in last step that is selection process. Crossover is acting on two parents and it is a binary genetic operator. 5) Mutation: As a result of crossover, the new generation introduced will only have the traits of the parents. This can sometimes lead to a problem where no new genetic material is introduced in the offspring. Mutation allows a new genetic pattern to be introduced in the new set of chromosomes. The selection process will retain it if the fitness of the mutated chromosome is higher than the general population, otherwise, selection will ensure that the chromosome does not live to mate in future. As with crossover, the mutation rate is defined to control how often mutation is applied [7].

\section{EE-LEACH MIMO SCHEME}

The sensor nodes in WSN are having with limited battery life so the main point of improvement of lifetime of wireless sensor networks directly focus on the factor of energy conservation. The networks based on clustering mainly divide the sensing area in the number of clusters and from each cluster one cluster head is selected. Other nodes in the cluster are called as cluster members. LEACH that is first energy efficient protocol used in WSN improves the life time of the network efficiently. This is a clustering based approach. With the number of advantages LEACH protocol also comes with some disadvantages like while choosing cluster head this protocol does not take into account the residual energy of the sensor nodes and also the cluster head distribution is non uniform. The EE-LEACH MIMO scheme provides an improvement over the LEACH protocol. 
In this scheme the network is divided into sectors of equal angles and the residual energy of sensor nodes also considered while choosing cluster head and cooperative nodes for MIMO system. The clustering is done only for one time. The network is divided into clusters by cutting it from center using an angle of $2 \pi / K_{\text {opt }}$. Sink inform the nodes to join the cluster nearest to them. The value of $K_{\text {opt }}$ is 5 for implementation of EE-LEACH MIMO scheme. All the operations are managed in rounds. For each round the selection of cluster head and cooperative nodes takes place.

\section{PROPOSED MODEL}

As EE-LEACH MIMO scheme is an advancement of LEACH. But there is a problem in this method. The network is partitioned at the equal angles. But as the deployment of sensor nodes is random in wireless sensor networks. So by dividing the network at equal angles, the nodes in each cluster will be different in number. May be there will exists a cluster with only 5 nodes and one cluster with 37 nodes. Then in this case the cluster head of the cluster with 5 nodes only will stay alive for more time as compared to the cluster head of a cluster having with 37 nodes. This is because the load on the cluster head of a cluster with only 5 nodes will be very less as compared to the load of cluster head of a cluster having with 37 nodes.

Our proposed algorithm divides the network to form the clusters in such a way or at such angles so that the number of nodes in each cluster should almost same. So that load on the cluster heads in each cluster should same. By doing this the life time of wireless sensor network can be enhanced. For achieve this goal, genetic algorithm is used.

$>$ Firstly the sensor nodes are deployed over the sensing field.

$>\quad$ Then the clustering is done by cutting the sensing field from center at equal angles by using parameter $2 \pi / K_{\text {opt }}$. Where $K_{\text {opt }}=5$.

$>$ Now the number of sensor nodes according to their position in sensing field is calculated in each cluster.

$>$ In order to have the same number of sensor nodes in each cluster, GA is implemented.

$>$ By implementing the GA the new angles are achieved in such a way that the number of sensor nodes at these new angles will be almost same in each cluster.

$>$ After that inter cluster and then from cluster heads to sink, transmission takes place.

\subsection{Energy model}

In order to study the energy efficiency of our scheme, we introduce the energy consumption model. The energy consumption for transmitting and receiving $l$-bit message in a distance of $d$ meters are respectively:

$$
\begin{aligned}
& E_{\text {tx_con }}=l *\left(\left(E_{\text {tx_elec }}\right)+\left(E_{\text {amp }} * d^{R}\right)\right) \\
& E_{\text {rx_con }}=l *\left(E_{\text {rx_elec }}\right)
\end{aligned}
$$

Where $l$ is the packet size and $E_{\text {tx_elec }}$ and $E_{\text {rx_con }}$ denotes the transmitter and receiver circuit energy consumption per bit. $E_{a m p}$, accounts for the effect of amplifier.
The energy of network is mainly consumed in: 1) data transmission from cluster member nodes to the cluster head. 2) Data transmission from the cluster head to the

sink.3) Data fusing in the cluster head.

\subsection{Fitness value}

By analyzing the problem we can find out a function by using which, the problem can be solved. But in some cases to find out the mathematical solution of such functions is not possible. To solve such problems GA is one of the optimize algorithm that can be used to find out the solution. In GA the particular function which we get by analyzing the problem is called as nutrient function. The value of this function is defined as the fitness value in GA.

\section{SIMULATION AND ANALYSIS}

This section contains the obtained various simulations results using the proposed scheme. The simulation results are obtained by using MATLAB software. Then the results of proposed algorithm are compared with the results of EE-LEACH MIMO scheme. The simulation results are obtained on the bases of the number of rounds. We use the number of rounds before the first node dies (FND), half nodes die (HND) and last node dies (LND) for evaluating the network lifetime.

\subsection{Simulation system papameters}

In the proposed scheme 100 sensor nodes are deployed. The sensor nodes are deployed in random order. These sensor nodes are having with the same initial energy. Each node can transmit $l$ bits of data in each round.

Table 1. System parameters

\begin{tabular}{|l|l|}
\hline Parameter & Value \\
\hline Area of network & $100 \mathrm{~m}^{*} 100 \mathrm{~m}$ \\
\hline Number of nodes & 100 \\
\hline Position of sink & $(50,175)$ \\
\hline$E_{\text {tx_con }}$ & $50 \mathrm{~nJ} / \mathrm{bit}$ \\
\hline$E_{\text {amp }}$ & $100 \mathrm{pJ} / \mathrm{bit} / \mathrm{m}^{2}$ \\
\hline Packet size & $2000 \mathrm{bits}$ \\
\hline Initial energy & $0.5 \mathrm{~J}$ \\
\hline$E_{\text {rx_con }}$ & $50 \mathrm{~nJ} / \mathrm{bit}$ \\
\hline Energy for data fusing & $5 \mathrm{~nJ} / \mathrm{bit}$ \\
\hline
\end{tabular}

\subsection{Simulation and analysis}

In the sensing field of area $100 \mathrm{~m}^{*} 100 \mathrm{~m}, 100$ sensor nodes are deployed. These sensor nodes are deployed randomly and represented in red color. Sink node is placed at a position $(50,175)$ and represented in blue color. The clustering is shown by using the lines in gray color. The randomly deployment of sensor nodes is shown in the figure given below: 


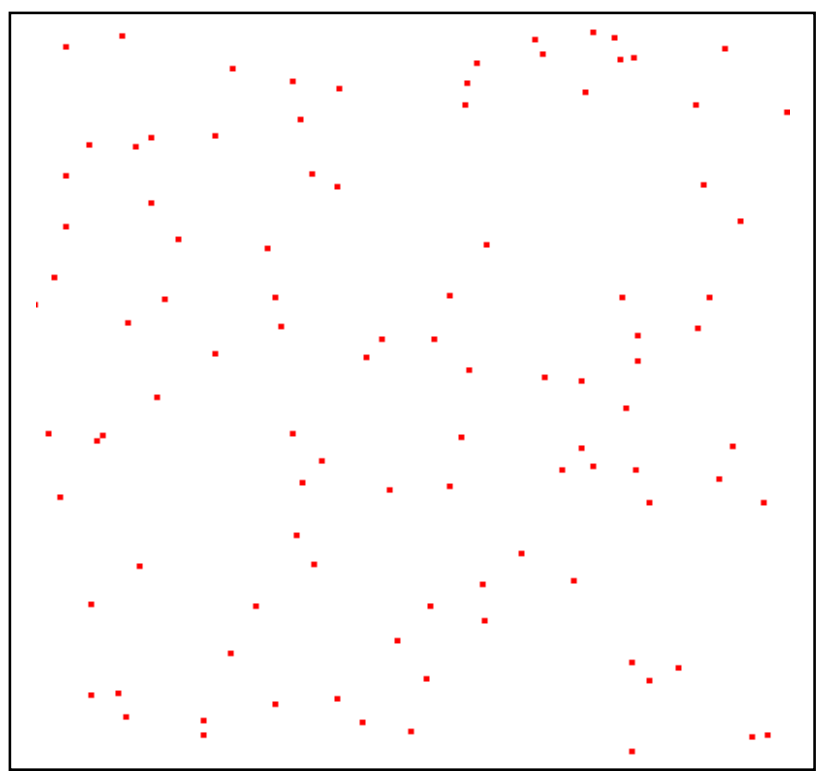

Fig.3. Randomly deployment of sensor nodes

Clustering done by using parameter $2 \pi / K_{\text {opt }}$ is shown as follow:

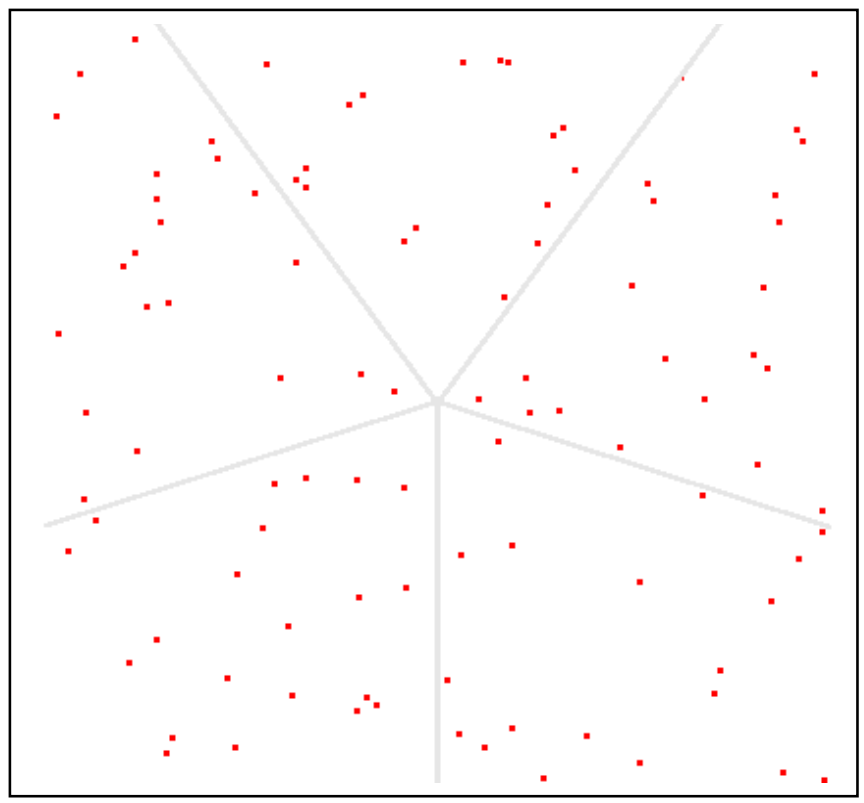

Fig.4. Clustering with equal angles $\left(2 \pi / K_{\text {opt }}\right)$

After implementing GA, new angles are achieved in order to make total number of sensor nodes almost equal in each cluster. This is also shown in figure given below:

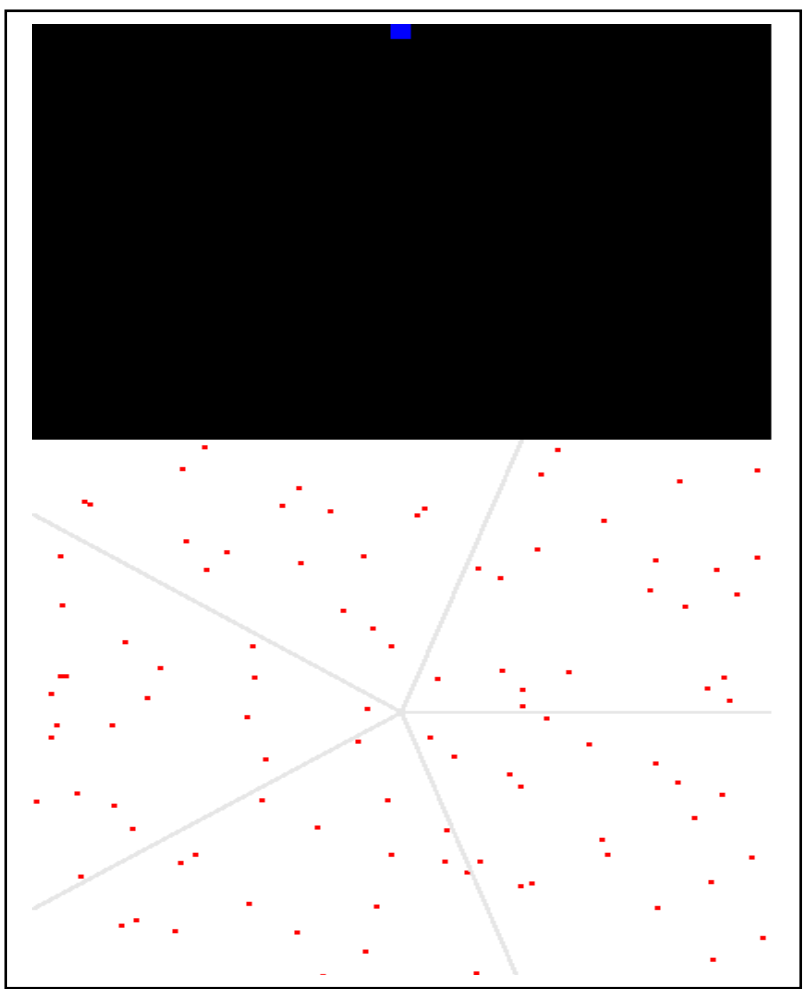

Fig.5. Clustering with new angles (optimized angles)

The simulation results of the implementing technique are show below in the graph:

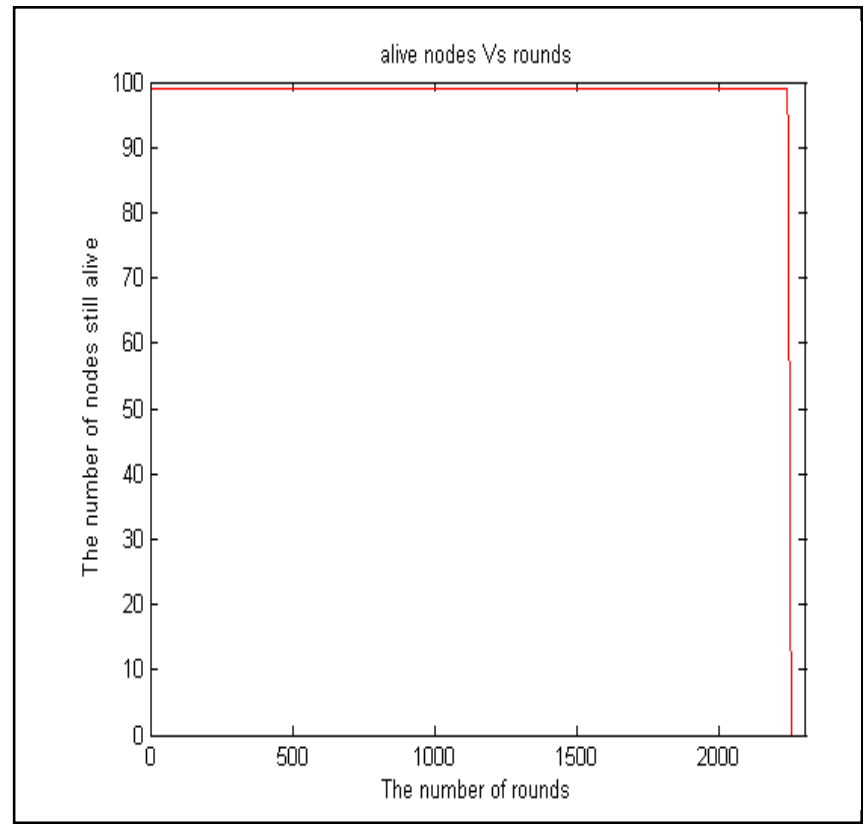

Fig.6. Total number of alive nodes with rounds

A comparison for the first node die (FND), half node die (HND) and last node die (LND) is shown in Table 2. On the bases of this the purposed algorithm and EE-LEACH MIMO algorithm is compared. 
Table 2. FND, HND and LND comparison

\begin{tabular}{|l|l|l|l|}
\hline & FND & HND & LND \\
\hline EE-LEACH MIMO & 1040 & 1274 & 1342 \\
\hline PROPOSED ALGO & 2230 & 2243 & 2261 \\
\hline
\end{tabular}

The above table shows that the first node, half nodes and last node dies later in purposed algorithm as compared to the EE-LEACH MIMO scheme. So because of this difference the lifetime of network is improved as compared to EE-LEACH MIMO scheme. In EE-LEACH MIMO, corresponding curve declines slowly and smoothly. It means that energy consumption among nodes in the network has not well balanced. But in proposed algorithm few nodes dies at the same time. Its curve is extremely steep. All of its nodes use out at almost a moment. That means the energy consumption among nodes in the network is well balanced. So the lifetime of the network using proposed algorithm is improved as compared to the EE-LEACH MIMO scheme.

\section{CONCLUSION}

In the paper, we proposed an optimized scheme, which uses the genetic algorithm for optimizing the network lifetime By comparison of our proposed scheme with EE-LEACH MIMO scheme is done on the bases of FND, HND and LND. It is seen that FND, HND and LND of proposed scheme appear noticeable improvements, these improvements are respectively 53\% 43\% and $41 \%$.. In our proposed algorithm the energy consumption is more balanced as compared to the EE-LEACH-MIMO scheme. The simulation result shows that the network lifetime is improved in case of proposed scheme as compared to the EE-LEACH MIMO scheme.

\section{REFERENCES}

[1] Dahlila P. Dahnil, Yaswant P. Singh, Chin Kuan Ho "EnergyEfficient Cluster Formation in Heterogeneous Wireless Sensor Networks: A Comparative Study" ICACT2011

[2] Mahmoud Naghibzadeh Peyman Neamatollahi, Hoda Taheri "A Hybrid Clustering Approach for Prolonging Lifetime in Wireless Sensor Networks” FEB 2011.

[3] Vivek Katiyar, Narottam Chand, Surender Soni "Improving Lifetime of Large-scale Wireless Sensor Networks through Heterogeneity" C2011 IEEE.

[4] M. Bani Yassein, A. Al-zou'bi, Y. Khamayseh, W. Mardini "Improvement on LEACH Protocol of Wireless Sensor Network (VLEACH)" International Journal of Digital Content Technology and its Applications Volume 3, Number 2, June 2009 .

[5] Jia Xu,Ning Jin, Xizhong Lou,Ting Peng,Qian Zhou, Yanmin Chen "Improvement of LEACH protocol for WSN" 2012 IEEE.

[6] Chenmin Li , Guoping Tan , Jingyu Wu, Zhen Zhang, Lizhong $\mathrm{Xu}$ "Analyzing Cluster-head Selection Mechanisms and Improving the LEACH" 2011 IEEE.

[7] Sajid Hussain, Abdul Wasey Matin, Obidul Islam "Genetic Algorithm for Hierarchical Wireless

Sensor Networks" JOURNAL OF NETWORKS, VOL. 2, NO. $\quad$ S, SEPTEMBER 2007 